\title{
Intrinsic transverse field in frustrated quantum Ising magnets: Physical origin and quantum effects
}

\author{
Gang Chen (1) \\ Department of Physics and Center of Theoretical and Computational Physics, The University of Hong Kong, Hong Kong, China
}

(Received 2 August 2019; published 3 December 2019)

\begin{abstract}
Transverse field Ising model is a common model in quantum magnetism and is often illustrated as an example for quantum phase transition. Its physical origin in quantum magnets, however, is actually not quite well-understood. The quantum mechanical properties of this model on frustrated systems are not well-understood either. We here clarify the physical origin, both extrinsic one and intrinsic one, for the transverse field of the quantum Ising model, and then explain the quantum effects in the kagome system. We discuss the quantum plaquette order and the quantum phase transition out of this ordered state in the rare-earth kagome magnets. Our specific results can find their relevance in the rare-earth tripod kagome magnets.
\end{abstract}

DOI: 10.1103/PhysRevResearch.1.033141

\section{INTRODUCTION}

The classical Ising model is a textbook model in the field of magnetism and statistical physics. The exact solution by Lars Onsager for the two-dimensional Ising model is a milestone of modern statistical physics and proved the very existence of continuous phase transitions with only shortrange interactions [1]. The far-reaching impact of Onsager's solution goes much beyond the original motivation [2,3]. Its quantum extension, the transverse field Ising model, contains the ingredient of the quantum phase transition and emergent low-energy quantum field theories at the criticality [4]. For the unfrustrated Ising interaction on systems like a square lattice, the transverse field Ising model can be well-understood from the high-dimensional classical Ising model and its thermal transition. On the frustrated systems, however, new ingredients may arise from the interplay between the quantum fluctuation and the geometrical frustration of the underlying lattices.

Besides the interesting physical properties of the transverse field Ising models, the physical origin of the transverse field Ising models is actually not well understood. This is related to the physical realization of this simple and important model. The Ising model requires a strong spin anisotropy in the spin space, and this almost immediately implies that, the magnetic system must have a strong spin-orbit coupling. Indeed, the localized moments of the proposed Ising magnets, such as quasi- $1 d$ magnets $\mathrm{CoNb}_{2} \mathrm{O}_{6}, \mathrm{BaCo}_{2} \mathrm{~V}_{2} \mathrm{O}_{8}, \mathrm{SrCo}_{2} \mathrm{~V}_{2} \mathrm{O}_{8}$, and various $2 d / 3 d$ rare-earth magnets, do arise from the strong spin-orbit entanglement, and the local moments have a strong orbital character [5-12]. In the case of the $\mathrm{Co}^{2+}$ local moment, the ion has a $3 d^{7}$ electron configuration and has one hole in the lower $t_{2 g}$ shell, and the spin-orbit coupling is active here. As a

Published by the American Physical Society under the terms of the Creative Commons Attribution 4.0 International license. Further distribution of this work must maintain attribution to the author(s) and the published article's title, journal citation, and DOI. result, the ion has a total $\operatorname{spin} S=3 / 2$ and an effective orbital angular momentum $L=1$, and the resulting total moment is given by the spin-orbit-entangled Kramers doublet. Because of the involvement of the orbital degrees of freedom, the exchange interaction between the Kramers doublet has to be anisotropic. This is indeed the underlying driving force for the Kitaev interactions in the Co-based honeycomb magnets $\mathrm{Na}_{2} \mathrm{Co}_{2} \mathrm{TeO}_{6}$ and $\mathrm{Na}_{3} \mathrm{Co}_{2} \mathrm{SbO}_{6}[13,14]$, and the anisotropic interaction in the pyrochlore cobaltate $\mathrm{NaCaCo}_{2} \mathrm{~F}_{7}$ [15-17]. For the case of quasi-1d magnets $\mathrm{CoNb}_{2} \mathrm{O}_{6}, \mathrm{BaCo}_{2} \mathrm{~V}_{2} \mathrm{O}_{8}$, and $\mathrm{SrCo}_{2} \mathrm{~V}_{2} \mathrm{O}_{8}$, because of the local $\mathrm{Co}^{2+}$ environment and the special lattice geometry, the system realizes the Ising interactions between the local moments. The transverse field is then introduced externally by applying a magnetic field normal to the Ising spin direction. This is feasible because the $\mathrm{Co}^{2+}$ local moment is a Kramers doublet and all the three components of the moments are magnetic. This is the external origin of the transverse field.

Is there an intrinsic origin of the transverse field? Our successful modeling [20] of the intertwined multipolar physics in the triangular lattice magnet $\mathrm{TmMgGaO}_{4}[18,19]$ suggests a positive answer. We start from our early understanding about the $\mathrm{Tm}^{3+}$ ion in $\mathrm{TmMgGaO}_{4}$ and then give an answer for the general cases. The $4 f$ electrons of the $\mathrm{Tm}^{3+}$ ion has a total spin $S=1$ and orbital angular momentum $L=5$, then the

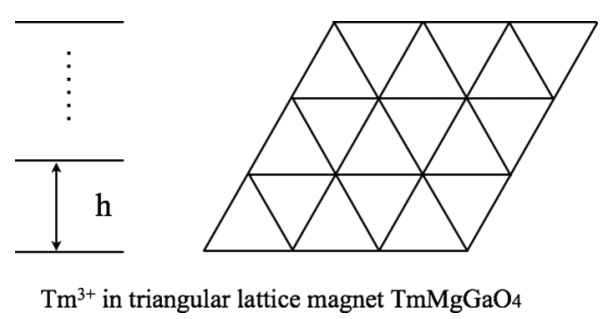

FIG. 1. The $\mathrm{Tm}^{3+}$ magnetic ions in $\mathrm{TmMgGaO}_{4}$ form a triangular lattice [18-20]. The lowest two crystal field singlets can be modelled as an effective spin-1/2 degree of freedom, and the weak crystal field splitting is modelled as a transverse field. This aspect of microscopics and physical model have been clarified in Ref. [20]. 
spin-orbit coupling leads to a total moment $J=6$. As we show in Fig. 1, the two lowest crystal field states of the $\mathrm{Tm}^{3+}$ ion are two singlets and they comprise two independent singlet irreducible representations of the $\mathrm{D}_{3 d}$ point group rather than one single two-dimensional irreducible representation. Because the total moment, $J$, is integer in nature, there is no Kramers theorem, and there is always a crystal field energy gap separating these two singlets [20]. This differs fundamentally from the usual non-Kramers doublet whose degeneracy is protected by the two-dimensional irreducible representation of the $\mathrm{D}_{3 d}$ point group. The two singlet wave functions primarily involve the $J^{z}= \pm 6$ components, and the group representation forbids $J^{z}= \pm 1$ here [20]. If one defines a pseudospin-1/2 degree of freedom that operates on these two singlets, only the $z$ component of the pseudospin carries the magnetic dipole moment, and the remaining two components are high order multipole moments that are related to high order products of $J$ moments. Because of this very unique property, the external magnetic field only acts on the $z$ component of the pseudospin, regardless of the direction of the external magnetic field. Thus the transverse field cannot be generated externally. Remarkably, our mother nature builds the transverse field intrinsically in this material [20] and brings quantum phenomena within itself. The observation is from the weak crystal field splitting that was modelled by us as the transverse field on the transverse multipolar component of the pseudospin [20].

What can we learn from the successful and special example of $\mathrm{TmMgGaO}_{4}$ ? The two weakly separated crystal field singlets of the $\mathrm{Tm}^{3+}$ ions are the consequences of the singlet representations of the point group and the non-Kramers nature of the ion. More generally, the point group symmetry of other rare-earth magnets is not as high as the $\mathrm{D}_{3 d}$ point group, and thus it is more common to have the singlet crystal field states for non-Kramers ions with integer spins. Therefore, in the rare-earth magnets with a low point group symmetry, if the two lowest singlets are well separated from the higher excited crystal field states, then we can single them out and build up a pseudospin-1/2 degree of freedom. This pseudospin- $1 / 2$ degrees of freedom will be responsible for the low-temperature magnetic properties. Like our case for the $\mathrm{Tm}^{3+}$ ion, the longitudinal component of the pseudospin is the dipolelike, and the transverse component is the multipolelike. If the terms of the wave functions for the two singlets are far apart in the $J^{z}$ basis, then the exchange interaction will be mostly Ising-like because it is a bit difficult for the system to flip " $J$ " multiple times during the superexchange process. More importantly, the remaining splitting between the two singlets could be modelled as the transverse field on the multipole component [20].

The above discussion clarifies an intrinsic origin for the transverse field of the transverse field Ising model that can potentially be relevant for many rare-earth magnets. To further explore the quantum properties on the frustrated system, we turn to a specific frustrated lattice that is kagome lattice. Apparently, the tripod rare-earth kagome magnets have already existed [21-24]. Moreover, the rare-earth kagome magnets can be obtained from the rare-earth pyrochlore magnets from the dimensional reduction by applying an external magnetic field along the [111] crystallographic direction that polarizes one sublattice [25-27]. For a kagome lattice, the twofold degenerate non-Kramers doublet is not allowed by the lattice symmetry, and there should always be a splitting between two lowest crystal field states. This naturally provides the transverse field. The major parts of this paper are to probe the existence and explore the experimental consequences of this intrinsic transverse field. Due to the strong geometrical frustration, the pure transverse field Ising model with an antiferromagnetic Ising interaction on the kagome lattice is known to have no transition throughout all parameter regime [28], and the system is smoothly connected to the polarized state in the strong transverse field limit. To create more structures from the geometrical frustration and the transverse field, we apply an external magnetic field to the system and establish a phase diagram with a phase transition. We show that, at the weak transverse field regime, the system develops a quantum plaquette order. There can be a direct quantum phase transition from this quantum plaquette order to the disordered state. We explain the dynamical properties in different regime and discuss some key experimental consequences.

The remaining parts of the paper are organized as follows. In Sec. II, we introduce our physical model and justify our introduction of the external magnetic field. In Sec. III, we carry out the perturbative treatment in the Ising limit and establish the quantum plaquette order. In Sec. IV, we regard the quantum plaquette ordered state as the parent state and demonstrate the relation between this state and the disordered state. We show that, the quantum plaquette order can be regarded as a confining phase of the compact U(1) gauge theory and the disordered state can be regarded as the Higgs phase. In Sec. V, we explain the thermodynamic properties and establish the excitations inside the disordered phase, and this excitation spectrum would be a strong evidence for the intrinsic transverse field in the system and the multipolar structure of the local moments. Finally, in Sec. VI, we conclude with a discussion of the experimental relevance and consequences.

\section{KAGOME LATTICE TRANSVERSE FIELD ISING MODEL}

We start with a brief introduction of the rare-earth tripod kagome magnet $A_{2} \mathrm{RE}_{3} \mathrm{Sb}_{3} \mathrm{O}_{14}$ where $A=\mathrm{Mg}, \mathrm{Zn}$ and $\mathrm{RE}$ refers to the rare-earth atom $(\mathrm{Pr}, \mathrm{Nd}, \mathrm{Gd}, \mathrm{Tb}, \mathrm{Dy}, \mathrm{Ho}, \mathrm{Er}$, $\mathrm{Yb}$, and Tm) [21-24]. Various interesting phases and results have already been suggested for this new family of materials. The $\mathrm{Nd}^{3+}, \mathrm{Dy}^{3+}, \mathrm{Er}^{3+}$, and $\mathrm{Yb}^{3+}$ ions have odd number of electrons per ion and thus support a Kramers doublet locally. For these Kramers doublet local moments, if the Ising spin is realized, the transverse field has to be generated externally. The $\mathrm{Gd}^{3+}$ ion is special and has a half-filled $4 f$ shell with a total spin $S=7 / 2$ and a quenched orbital angular momentum, the atomic spin-orbit coupling is inactive for this ion. The remaining ones all have integer total moments. Unlike the rare-earth pyrochlore magnets where non-Kramers doublets exist in many compounds, there is no such non-Kramers doublet in the tripod kagome magnets. The symmetry of the kagome lattice is too low to support a twofold non-Kramers degeneracy. There is always a finite crystal field splitting between the would-be non-Kramers doublet. Similar to the context of the pyrochlore magnets, we can still introduce an effective spin-1/2 degree of freedom here except that we 
need to introduce a transverse field to take care of the crystal field splitting between the two singlets of the non-Kramers doublets. Like the case for the $\mathrm{Tm}^{3+}$ ion in $\mathrm{TmMgGaO}_{4}$, the transverse field is of intrinsic origin. The resulting model is given as

$$
H=\sum_{i j}\left[J_{i j} S_{i}^{z} S_{j}^{z}+\cdots\right]-\sum_{i} h S_{i}^{x},
$$

where "..." refers to the XY-like spin flipping term $\left(S_{i}^{+} S_{j}^{-}\right)$ and the pair-flipping term $\left(S_{i}^{+} S_{j}^{+}\right)$that can be written down from the symmetry analysis, and these extra terms would have a strong bond dependence and bring the quantum fluctuations to the Ising part. This kind of anisotropic spin model has been widely studied in the context of the rare-earth pyrochlores and the triangular lattice spin liquid materials. This anistropic interaction on the kagome lattice has not yet been discussed in the literature and we will address it in an another paper. Here, since the intrinsic transverse field already brings quantum properties into the system, we will focus on this transverse field Ising model. From the materials' point of view, we have learned from the experience of the rare-earth pyrochlore magnets that some materials such as $\mathrm{Ho}_{2} \mathrm{Ti}_{2} \mathrm{O}_{7}$ do behave quite Ising-like [29]. It is natural to expect that, in the tripod kagome system, this Ising feature could persist. Indeed, it was proposed that the Dy-based and Ho-based tripod kagome magnets do behave Ising-like where the intrinsic transverse field was indicated for the Ho-based one [30].

The ferromagnetic quantum Ising model does not lead to any unknown properties even on this frustrated lattice. Thus we consider an antiferromagnetic Ising interaction and restrict ourselves to the nearest neighbors. It was actually studied numerically long time ago that the transverse field Ising model on the kagome lattice has neither phase transition nor symmetry breaking. The system remains disordered throughout the parameter space [28]. This was referred as "disorder-bydisorder" [28], in contrast to the "order-by-disorder" [31,32] for the transverse field Ising model on the triangular lattice in the weak field regime [31-34]. The essential reason for the disordered state even in the weak field regime arises from the fact that the up-up-down and down-down-up triangular plaquettes are degenerate and both of them appear in the low-energy manifold. To create more structures to the phase diagram, we here apply an external magnetic field to the system. Only the dipolar component $\left(S^{z}\right)$ of the pseudospin will couple to the external magnetic field regardless to the orientation of the external magnetic field. This field could have the effect of removing half of the active spin configurations in each triangular plaquette. So our model now becomes

$$
H=\sum_{\langle i j\rangle} J S_{i}^{z} S_{j}^{z}-\sum_{i} h S_{i}^{x}-\sum_{i} B S_{i}^{z},
$$

where only the nearest-neighbor interaction is considered. In the absence of the intrinsic transverse field $h$, an infinitesimal external magnetic field $B$ would already select all up-up-down spin configuration and create a magnetization plateau. This magnetization plateau persists up to the field value of $J / 2$ without extra interactions. This plateau regime in $B \in(0, J / 2)$ is identical to the "kagome spin ice" that was obtained from the classical pyrochlore spin ice by applying a magnetic field

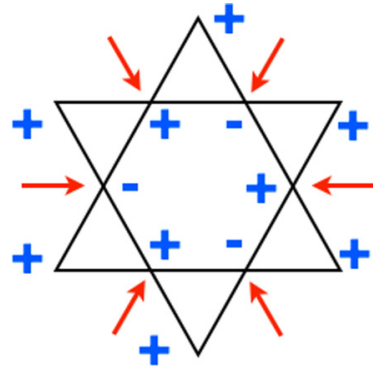

(a)

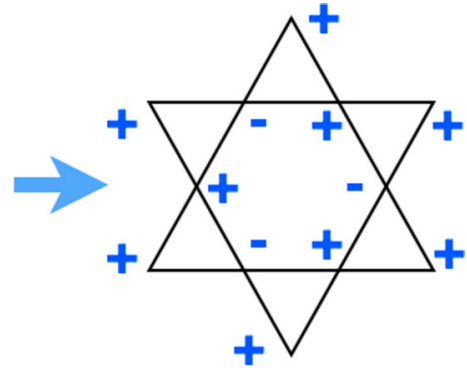

(b)
FIG. 2. The sixth order degenerate perturbation process from the intrinsic transverse field within the degenerate "up-up-down" spin configurations. The red arrows indicate the application of the transverse field on this site. "+" refers to spin-up, and "-" refers to spin-down. See the main text for the detailed discussion.

along the [111] direction to polarize one sublattice [25,26,35]. However, this is classical physics. In the following section, we analyze the quantum effect of the intrinsic transverse field within this degenerate spin manifold. Some aspects of this model such as the ordered structure have been established in the early numerical study [31,32]. Our purpose is to propose this model for the rare-earth kagome magnets with non-Kramers doublets. We further propose the fractionalized nature of the phase transition based on the lattice gauge theory, and explore the experimental consequences of different phases and the transition due to the multipolar nature of the local moments. The experimental signature of the intrinsic transverse field is emphasized.

\section{PERTURBATION THEORY AND QUANTUM PLAQUETTE ORDERS}

We continue to work within the degenerate "up-up-down" spin configurations due to our introduction of the external magnetic field on the dipolar component of the pseudospin. Once a weak transverse field is introduced, the extensive degeneracy will be lifted, and a degenerate perturbation theory is needed. The leading effect comes from the sixth order, that is depicted in Fig. 2, is summarized below,

$$
H_{6}=-J_{6} \sum_{\square} S_{1}^{x} S_{2}^{x} S_{3}^{x} S_{4}^{x} S_{5}^{x} S_{6}^{x},
$$

where $J_{6} \sim \mathcal{O}\left(h^{6} / J^{5}\right)>0$ and the "-" sign takes care of even number of perturbation series. The lower order perturbations either vanish or give a constant shift to the classical energies. Our sixth order effective Hamiltonian, $H_{6}$, operates on the degenerate manifold of "up-up-down" spin configuration. This process can be mapped to a quantum dimer model on the dual honeycomb lattice that is formed by connecting the centers of the triangular plaquettes if the down spin is mapped to the dimer convering on the bond connecting the centers of the neighboring triangular plaquettes (see Fig. 3). This is an exact mapping. The quantum dimer model is given as

$$
H_{6}=-J_{6} \sum_{\square}\left[\left|\square_{1}\right\rangle\left\langle\square_{2}|+| \square_{2}\right\rangle\left\langle\square_{1}\right|\right],
$$



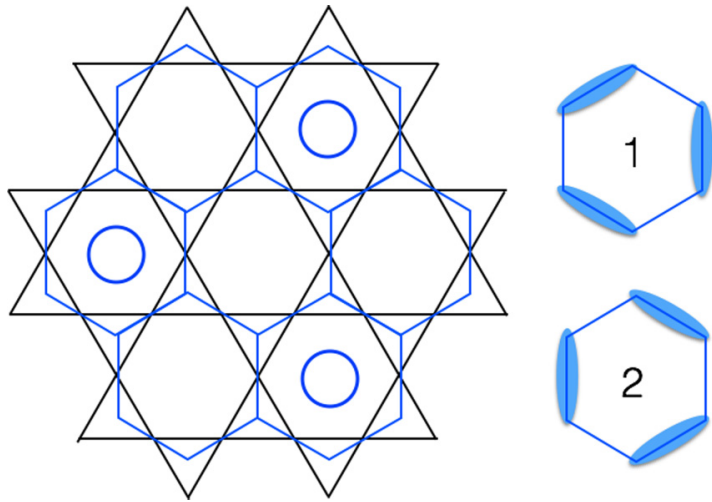

FIG. 3. The kagome lattice and its dual honeycomb lattice. Dimer configurations 1 and 2 refer to two distinct dimer coverings on the elementary hexagon. In the quantum plaquette ordered phase, the hexagons with blue circles refer to the resonating hexagon where the three dimers form a quantum linear superposition of the dimer configurations 1 and 2 .

where $\left|\square_{1}\right\rangle$ and $\left|\square_{2}\right\rangle$ refer to the two alternating dimer coverings on the elementary hexagons of the dual honeycomb lattice (see Fig. 3). This quantum dimer model is known to have a plaquette dimer order by breaking the lattice translation symmetry, and the unit cell has been tripled [36]. Returning back to the spin language, this plaquette dimer order corresponds to the quantum plaquette order where in the resonating hexagon with a blue cirlce (see Fig. 4) the ground state can be approximated as $(|\uparrow \downarrow \uparrow \downarrow \uparrow \downarrow\rangle+|\downarrow \uparrow \downarrow \uparrow \downarrow \uparrow\rangle) / \sqrt{2}$. We have listed the spin state for the six spins on the resonating hexagon. This is an even cat state of six spins on the hexagon.

How does one probe this quantum plaquette order? Thermodynamically, there should a finite temperature phase transition as one lowers the temperature. Moreover, this order breaks the lattice translation, and one should be able to observe the magnetic Bragg peaks at the wavevectors $( \pm 4 \pi / 3,0)$ where we have set the original lattice constant to unity. This corresponds to the momentum points $K$ and $K^{\prime}$ in Fig. 4. In this system, only $S^{z}$ is time reversally odd

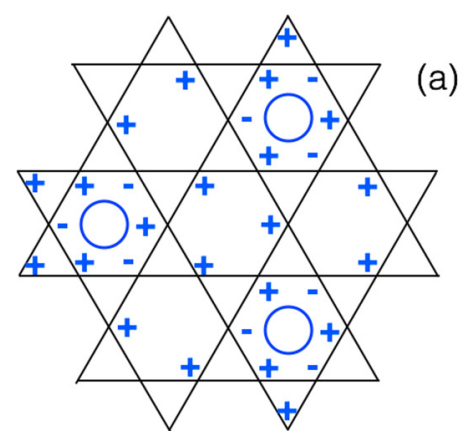

(b)

FIG. 4. The quantum plaquette order in terms of the spin configuration. On the resonating hexagon, the six spins form a quantum linear superposition of the alternating spin orientations. The ordering wave vectors of the quantum plaquette order occur at $K$ and $K^{\prime}$ points. The blue hexagon inside the big hexagon in (b) is the reduced Brillouin zone when the system develops the quantum plaquette order. and can be detected from the conventional neutron scattering measurements. So, we expect the magnetic Bragg peak to be observed in the $S^{z}-S^{z}$ correlator for an inelastic neutron scattering measurement. Nuclear magnetic resonance (NMR) measurement can also be a convenient probe of the number of internal fields that are generated by the enlarged $S^{z}$ magnetic unit cell from the quantum plaquette order.

How about the elementary excitations? Again, As only $S^{z}-S^{z}$ correlator is detectable, we analyze the physics content that is contained in this correlator. If the magnetic state only has $\left\langle S^{z}\right\rangle \neq 0$, the $S^{z}-S^{z}$ correlator can only detect two-magnon excitations and would not be able to observe coherent magnon excitations. Because of the transverse field, $\left\langle S^{x}\right\rangle \neq 0$ both on the ordered side and on the disordered side. Inside the quantum plaquette ordered state, the $S^{z}-S^{z}$ correlator will be able to observe the coherent magnon modes, as the $S^{z}$ operator creates spin-flipping events for the $S^{x}$ configurations and this corresponds to the coherent magnon creation. This is also the underlying reason that we can observe the coherent magnon excitations for the triangular lattice magnet $\mathrm{TmMgGaO}_{4}$ and make a reasonable comparison with the spin wave theory. This could persist to the disordered side, although the number of modes will be restored to the one without the translation symmetry breaking.

Finally, in contrast to the ordered state from the quantum order by disorder for the triangular lattice case [31], the quantum plaquette order for the kagome system is more complicated as it is a quantum entangled state within the enlarged unit cell, and the conventional spin wave theory fails. Although this quantum plaquette order would manifest itself as Bragg peaks in the neutron scattering measurements, its quantum nature distinguishes itself from other conventional magnetic orders. The magnetic excitation cannot be captured well by the spin wave theory that is based on the single site magnetic orders. To compute the magnetic excitation spectrum inside the quantum plaquette ordered phase in the future, one needs to first resolve the local energy states within the enlarged unit cell because of the entangled nature of the ground state and represent these states/operators with a restructured flavor wave theory.

\section{NONPERTURBATIVE TREATMENT}

Having established that our model has a quantum plaquette order in the weak intrinsic transverse field limit, we continue to understand the structure of this phase when the field is large. Certainly, in the strong field limit, the ground state is a trivial disordered state and all the spins try to align themselves with the transverse field. The natural questions are whether there exists other competing phase between these two phases in the two opposite limits. If there is no intermediate phase, what would be the quantum phase transition between these two phases? To address these questions, we first provide another perspective of the quantum plaquette order and then regard this state as a parent state. We will explore the instability of this state and see what states we can obtain.

It is well-known that the quantum dimer model on the bipartite lattice is described by the compact $\mathrm{U}(1)$ lattice gauge theory, so is the dimer model for our dual honeycomb lattice. For two spatial dimensions, this U(1) lattice gauge 
theory is confining due to the proliferation of the spacetime monopole events. Our quantum plaquette order is essentially the consequence of the confinement. The role of the transverse field in the quantum plaquette ordered state is to create the spinon-antispinon pair, allow the spinons to hop, and lower the energy of the spinons gradually, although the spinons are actually confined. Here the spinon/antispinon refers to the defect triangular plaquette that violates the up-up-down condition. Under this picture, eventually the spinon band gap vanishes and the spinons are condensed, the resulting phase would be a Higgs phase of the compact U(1) lattice gauge theory.

\section{A. Transition between the confinement and the Higgs phases}

To describe the transition out of the quantum plaquette ordered phase, we focus on the spinon matter and construct a mean-field description to trace the spinons. To access the transition, we ignore the confined nature of the spinons in the quantum plaquette ordered state. This should be appropriate at the transition where the translation symmetry is restored, but is not a good approximation in the quantum plaquette ordered phase as the translation symmetry is broken. We first recast the microscopic Hamiltonian into the following form:

$$
H=\sum_{\Delta_{r}} \frac{J}{2}\left(\sum_{i \in \Delta_{r}} S_{i}^{z}-\frac{1}{2}\right)^{2}-h \sum_{i} S_{i}^{x},
$$

where the first term takes care of the up-up-down condition from the introduction of the external field on the dipolar component $S^{z}$, and " $\triangle_{r}$ " refers to the triangular plaquette that is centered at $\boldsymbol{r}$. As it is known from Fig. 3, the centers of the triangular plaquettes on the kagome lattice form a honeycomb lattice. We here introduce the spinon operators in the spirit similar to the one used in the context of pyrochlore spin ice system [37],

$$
\begin{aligned}
S_{i}^{+} & =\Phi_{r}^{\dagger} \Phi_{r^{\prime}} s_{r r^{\prime}}^{+}, \\
Q_{r} & =\eta_{r}\left(\sum_{i \in \Delta_{r}} S_{i}^{z}-\frac{1}{2}\right),
\end{aligned}
$$

where $S_{i}^{x}=\left(S_{i}^{+}+S_{i}^{-}\right) / 2$, and the site $i$ is the shared site of two neighbor triangular plaquettes at $\boldsymbol{r}$ and $\boldsymbol{r}^{\prime}$. Here, we choose $r$ to be in the I sublattice of the honeycomb lattice and $\boldsymbol{r}^{\prime}$ to be in the II sublattice of the honeycomb lattice, $s_{r r^{\prime}}^{+}$ is the $\mathrm{U}(1)$ gauge link variable, and $Q_{r}$ counts the spinon number density with $\eta_{r}= \pm 1$ for I/II sublattice. $\Phi_{r}^{\dagger}\left(\Phi_{r}\right)$ is the creation (annihilation) operator for the spinon at $r$. We have the commutation relations

$$
\begin{gathered}
{\left[\Phi_{r}, Q_{r^{\prime}}\right]=\Phi_{r} \delta_{r r^{\prime}},} \\
{\left[\Phi_{r}^{\dagger}, Q_{r^{\prime}}\right]=-\Phi_{r}^{\dagger} \delta_{r r^{\prime}} .}
\end{gathered}
$$

Under the parton construction, the physical Hilbert space of the spins is enlarged to the ones by $\Phi_{r}, Q_{r}$ and the gauge link. Once the Hilbert space constraint is imposed, the physical
Hilbert space is restored [37]. With this transformation, the physical Hamiltonian can be expressed as

$$
H=\sum_{r} \frac{J}{2} Q_{r}^{2}-\frac{h}{2} \sum_{\left\langle r r^{\prime}\right\rangle}\left[\Phi_{r}^{\dagger} \Phi_{r^{\prime}} s_{r r^{\prime}}^{+}+\text {H.c. }\right] .
$$

This model then describes the spinon hopping on the dual honeycomb lattice that is minimally coupled with the $\mathrm{U}(1)$ gauge link. The first term of the above Hamiltonian is the energy penalty that constrains the spinon density fluctuations. To solve this model, we carry out the standard gauge mean-field treatment and set $\Phi_{r}=e^{-\phi_{r}}$ with $\left[\phi_{r}, Q_{r^{\prime}}\right]=i \delta_{r r^{\prime}}$. From the knowledge of the previous sixth order perturbation calculation, the system would prefer a zero-flux sector for the spinons, and we can choose $s_{r r^{\prime}}^{ \pm}=1 / 2$ to fix the U(1) gauge link. Then, under the coherent state path integral for the bosonic spinons, the dispersions of the spinons can be established and are given by

$$
\omega_{ \pm}(\boldsymbol{k})=\left[2 J\left(\lambda \pm \frac{h}{4}\left|\sum_{\left\{\boldsymbol{b}_{i}\right\}} e^{i \boldsymbol{k} \cdot \boldsymbol{b}_{i}}\right|\right)\right]^{\frac{1}{2}},
$$

where $\lambda$ is the lagrangian multiplier to fix the unimodular condition for $\left|\Phi_{r}\right|=1$ and the spinon is condensed at the $\Gamma$ point. In addition, $\left\{\boldsymbol{b}_{i}\right\}$ is the set of three nearest-neighbor bonds of the dual honeycomb lattice. As the spinon is condensed at the $\Gamma$ point, the resulting spin state is a disordered state with a finite and uniform $\left\langle S^{x}\right\rangle$, i.e.,

$$
\left\langle S_{i}^{x}\right\rangle=\frac{1}{2}\left[s_{r r^{\prime}}^{+}\left\langle\Phi_{r}^{\dagger}\right\rangle\left\langle\Phi_{r^{\prime}}\right\rangle+\text { H.c. }\right],
$$

where the expectation is taken with respect to the spinon condensate at the $\Gamma$ point. The above description would suggest a direct transition from the confining phase to the Higgs phase, and the numerical calculation finds the critical point occurs at $h_{c}=1.26 \mathrm{~J}$. This transition is continuous at the mean-field level and may become weakly first order when the low-energy gauge fluctuation is included [38].

\section{B. Fractionalization and continuum at the criticality}

To probe the possible existence of the fractionalization at the transition or at least above the energy scale where our mean-field theory would work, we suggest the meansurement of the $S^{+}-S^{-}$spin correlation function. This spin correlator contains the information about the spinon dynamics. It corresponds to the creation of the spinon-antispinon, the evolution of them in space and time, and eventually, the annihilation of them. Thus the $S^{+} S^{-}$correlator would detect the 2-spinon continuum. Although this correlator is not directly measurable in the inelastic neutron scattering measurement for the nonKramers doublet local moments, as this model does not have the sign problem for the quantum Monte Carlo simulation, it can be useful to probe this correlator in the future numerical simulation. For the triangular lattice, this model has been extensively simulated [31-34] where the dynamical correlation properties and effect of long-range dipolar interaction were numerically studied recently $[39,40]$. On the triangular lattice, the system does not have exotic ground state and the physics is well captured within the conventional symmetry breaking $[32,33]$ and the associated spin wave like quasiparticle picture 


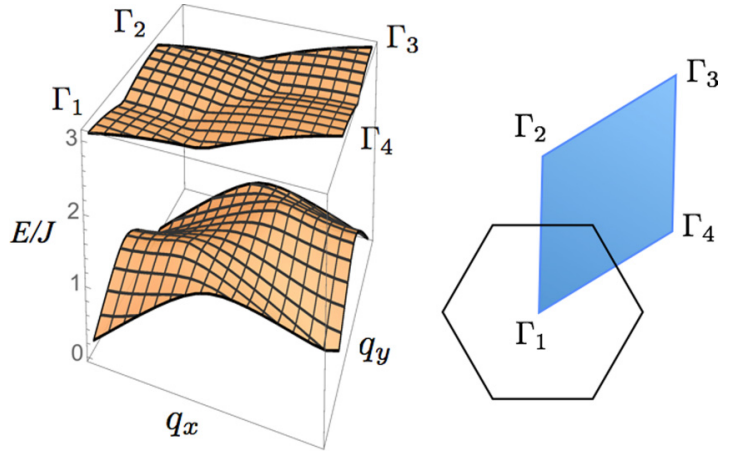

FIG. 5. The lower and upper excitation edges of the 2-spinon continuum at the phase transition. We plot the excitation continuum with respect to the parallelogram-shaped Brillouin zone. The continuum is gapless at the $\Gamma$ point. The right panel is the Brillouin zone with all four equivalent $\Gamma$ points marked.

[20]. In contrast, for our kagome system, we would encounter fractionalization [41-43]. Theoretically, one could obtain the structure of the 2-spinon continuum within the mean-field approach in the previous section. This is established by the energy-momentum conservation with

$$
\begin{aligned}
\boldsymbol{q} & =\boldsymbol{k}_{1}+\boldsymbol{k}_{2}, \\
E_{\boldsymbol{q}} & =\omega_{\mu}\left(\boldsymbol{k}_{1}\right)+\omega_{\nu}\left(\boldsymbol{k}_{2}\right),
\end{aligned}
$$

where $\boldsymbol{q}$ is the total crystal momentum and $E_{\boldsymbol{q}}$ is the total energy of the two spinons. Here $\mu$ and $v$ are the branch indices for the spinons and take \pm . The minima and maxima of $E_{\boldsymbol{q}}$ define the lower and the upper excitation edges of the 2-spinon continuum. The lower and upper edges are plotted in Fig. 5 using the mean-field parameters at the transition, and the continuum covers a large energy bandwidth. The mean-field theory qualitatively captures the fractionalized nature of the excitations at the transition.

\section{PHYSICAL PROPERTIES OF THE DISORDERED PHASE}

When the transverse field is large, the system will be in a disordered state with the Ising spin language. For the model in the absence of the external field, the transverse field Ising model on the kagome lattice remains disordered for all parameter range. From the experimental point of view, it would be quite useful to extract the parameters in the model. For this purpose, as we show below, the conventional thermodynamic measurements would be sufficient. Moreover, the unique multipolar structure of the local moments generates peculiar structures in the dynamic spin structure factor measurements.

\section{A. Thermodynamic properties}

As the external magnetic field only couples to the dipole component $S^{z}$, we now view the $B$ field as a probing field. The magnetization is nonvanishing only along the $z$ direction. Due to the intrinsic transverse field, the model does not have any continuous spin rotational symmetry. The magnetic susceptibility should simply be a constant in the zero temperature limit. This constant magnetic susceptibility can be obtained by a conventional self-consistent mean-field treatment with

$$
\begin{aligned}
H & =\sum_{\langle i j\rangle} J S_{i}^{z} S_{j}^{z}-h \sum_{i} S_{i}^{x}-B \sum_{i} S_{i}^{z} \\
& \rightarrow \sum_{\langle i j\rangle} J S_{i}^{z}\left\langle S_{j}^{z}\right\rangle-h \sum_{i} S_{i}^{x}-B \sum_{i} S_{i}^{z}
\end{aligned}
$$

where the expectation is taken with respect to the mean-field ground state. From the induced magnetization, it is ready to obtain the zero-temperature magnetic susceptibility,

$$
\chi_{0}=\left.\frac{1}{N} \sum_{i} \frac{\partial\left\langle S_{i}^{z}\right\rangle}{\partial B}\right|_{B \rightarrow 0}=\frac{1}{2 h+4 J},
$$

where $N$ is the total number of spins. Besides the susceptibility in the zero-temperature limit, the Curie-Weiss temperature from the high-temperature magnetic susceptibility provides another quantitative information with $\Theta_{\mathrm{CW}}^{z}=-J$ where only the $z$ component is meaningful. With $\chi_{0}$ and $\Theta_{\mathrm{CW}}^{z}$, it is sufficient to extract the couplings.

\section{B. Dynamic properties}

The magnetic excitations are measured by inelastic neutron scattering through the $S^{z}-S^{z}$ correlation. Here only $S^{z}-S^{z}$ correlation is contained in the inelastic neutron scattering spectrum because only $S^{z}$ is coupled to the neutron spin. In the disordered state, $\left\langle S^{x}\right\rangle$ is nonvanishing. As $S^{z}$ does not commute with $S^{x}$, what $S^{z}$ does is to flip $S^{x}$ and create coherent excitations. Thus $S^{z}-S^{z}$ correlation measures the coherent excitations with respect to the disordered state. This result really arises from the multipolar nature of the local moment. As the disordered state is smoothly connected to the finite temperature paramagnetic state, the coherent excitations, that are recorded in the $S^{z}-S^{z}$ correlation, would persist to the finite temperatures. Experimentally, this may be mysterious.

We consider the model with $B=0$, and set the spin wave transformation as

$$
\begin{aligned}
& S_{i}^{z}=\frac{1}{2 i}\left(b_{i}-b_{i}^{\dagger}\right), \\
& S_{i}^{x}=\frac{1}{2}-b_{i}^{\dagger} b_{i} .
\end{aligned}
$$

The linear spin wave Hamiltonian is then given as

$$
\begin{aligned}
H_{s w}= & \sum_{\boldsymbol{k}} \sum_{\mu} h b_{\boldsymbol{k} \mu}^{\dagger} b_{\boldsymbol{k} \mu}+\sum_{\boldsymbol{k}} \sum_{\mu \neq v} A_{\mu \nu}(\boldsymbol{k}) b_{\boldsymbol{k} \mu}^{\dagger} b_{\boldsymbol{k} v} \\
& +\sum_{\boldsymbol{k}} \sum_{\mu \neq v}\left[B_{\mu \nu}(\boldsymbol{k}) b_{\boldsymbol{k} \mu}^{\dagger} b_{-\boldsymbol{k} v}^{\dagger}+\text { H.c. }\right],
\end{aligned}
$$

where $\mu$ refers to the sublattices of the kagome lattice, and we have

$$
\begin{gathered}
A_{\mu \nu}(\boldsymbol{k})=\frac{J}{2} \cos \left(\boldsymbol{k} \cdot \boldsymbol{a}_{\mu \nu}\right), \\
B_{\mu \nu}(\boldsymbol{k})=-\frac{J}{4} \cos \left(\boldsymbol{k} \cdot \boldsymbol{a}_{\mu \nu}\right) .
\end{gathered}
$$




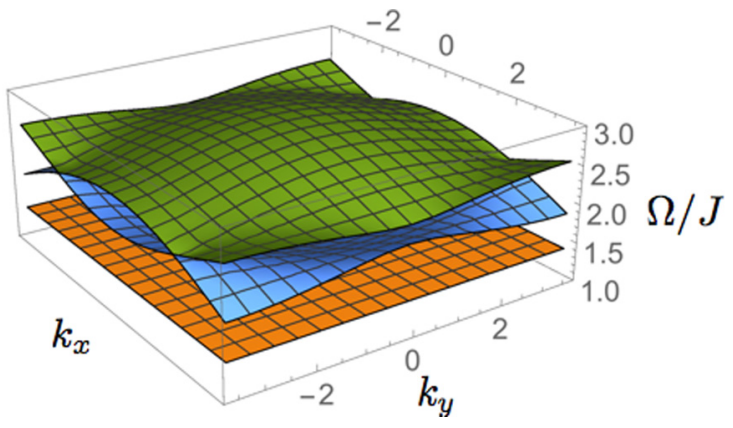

FIG. 6. The dispersions of the three magnetic excitations in the disordered phase. Here we set $h / J=2$. The lattice constant is set to unity.

Here, $\boldsymbol{a}_{\mu \nu}$ is the nearest-neighbor vector connecting sublattice $\mu$ and sublattice $v$ for the convention $\mu>v$.. The magnetic excitations have three branches with the dispersions,

$$
\begin{aligned}
& \Omega_{1}(\boldsymbol{k})=\left(h^{2}-h J\right)^{\frac{1}{2}}, \\
& \Omega_{2}(\boldsymbol{k})=\left[\frac{2 h^{2}+h J-h J\left[3+2 \sum_{\boldsymbol{a}_{\mu \nu}} \cos \left(2 \boldsymbol{k} \cdot \boldsymbol{a}_{\mu \nu}\right)\right]^{\frac{1}{2}}}{2}\right]^{\frac{1}{2}}, \\
& \Omega_{3}(\boldsymbol{k})=\left[\frac{2 h^{2}+h J+h J\left[3+2 \sum_{\boldsymbol{a}_{\mu \nu}} \cos \left(2 \boldsymbol{k} \cdot \boldsymbol{a}_{\mu \nu}\right)\right]^{\frac{1}{2}}}{2}\right]^{\frac{1}{2}},
\end{aligned}
$$

where the first band is a flat band. The dispersions are plotted in Fig. 6. The flat band here is not due to the frustration of the interaction and the lattice, but is from the fact that the exchange part only involves the exchange/hopping of $b_{i}-b_{i}^{\dagger}$ and does not involve $b_{i}+b_{i}^{\dagger}$. The latter is the origin of the flat dispersion.

\section{DISCUSSION}

In this discussion section, we will do three things. We first review the tripod kagome magnets with the rare-earth ions, and then discuss the kagome magnets from the rare-earth pyrochlore magnets via the dimensional reduction, and finally provide some perspectives about the transverse field Ising magnets on the frustrated lattices.

Most of the research about the tripod kagome magnets are experimental $[21,22,30]$. This is a bit different from the kagome lattice Heisenberg-like magnets such as the Herbertsmithite families where both theories and experiments are quite active [44]. It is thus beneficial to this new topic of kagome magnets if the theoretical inputs are provided. Due to some similiarity with the rare-earth pyrochlore and the rare-earth triangular lattice magnets, it would be interesting to explore the generic anisotropic spin model on the kagome lattice. The rare-earth tripod kagome magnets can be another platform to study the interplay between the spin-orbit entanglement and the geometrical frustration. Because this is a $2 d$ lattice with relatively lower symmetries, more spin interactions (beyond the ones for pyrochlore and triangular lattices) are allowed, and new magnetic orders and phases may thus be stabilized. While for the Kramers ion, the above expectation may be a simple and natural extension from other lattices. The tripod kagome magnets, however, do bring additional features for the non-Kramers ions. The low symmetry of the kagome lattice removes the non-Kramers doublets completely and splits them into multiple singlets. The physics that we have introduced in this paper is about the magnetically active lowest two singlets that can be approximately treated as an effective pseudospin-1/2 non-Kramers doublets with an intrinsic transverse field. The quantum plaquette orders and the phase transitions could be potentially tested in future experiments. Moreover, as along as the Ising spin condition is maintained, even in the presence of the weak transverse spin exchange interactions that could exist in real materials, our results in this paper will still hold.

It is well-known that the kagome lattice magnets can also be obtained from the rare-earth pyrochlore magnets by applying an external magnetic field along the [111] direction [26]. Due to the anisotropic coupling to the external magnetic field, one sublattice will be polarized. If the pyrochlore system is in the spin ice regime, the reduced kagome system would be in the kagome ice regime. The original nonKramers doublets of the pyrochlore magnets will remain to be non-Kramers doublets under this setting, and thus there is no intrinsic transverse field here. This, however, may not be the end of the story. To generate the intrinsic transverse field, one could grow the pyrochlore thin film along the [111] direction and place it on a substrate. The strain from the substrate will modify the lattice symmetry and remove the twofold degeneracy of the non-Kramers doublet. On the other hand, the system has the magnetoelastic coupling. This coupling was suggested by D. Khomskii to induce the electric dipole moment once the spin configuration is modified from the spin ice one [45]. The distortion of the lattice and/or the displacement of the ions will necessarily lower the lattice symmetry and generate a finite splitting among the non-Kramers doublet, and this can be treated as an intrinsic transverse field.

In this paper, we have delivered the frustrated quantum Ising model with an intrinsic transverse field on the kagome lattice. Since the mechanism for the intrinsic transverse field with low crystal symmetries can generally apply to the nonKramers ions, the quantum Ising model can be realized and explored among other frustrated rare-earth magnets such as the FCC double perovskites [46].

\section{ACKNOWLEDGMENTS}

We acknowledge Zhiling Dun, Martin Mourigal, Joseph Paddison, and Tao Xiang for discussion, and Cenke $\mathrm{Xu}$ and Yang Qi for a more recent discussion. This work is supported by the Ministry of Science and Technology of China with Grants No. 2016YFA0301001, No. 2016YFA0300501, No. 2018 YFE0103200 and by the General Research Fund (GRF) No. 17303819 from the Research Grants Council of Hong Kong. This work was performed in part at Aspen Center for Physics, which is supported by National Science Foundation Grant No. PHY-1607611. This work was partially supported by a grant from the Simons Foundation. 
[1] L. Onsager, Crystal statistics. I. A two-dimensional model with an order-disorder transition, Phys. Rev. 65, 117 (1944).

[2] B. Kaufman, Crystal statistics. II. Partition function evaluated by spinor analysis, Phys. Rev. 76, 1232 (1949).

[3] B. Kaufman and L. Onsager, Crystal statistics. III. Short-range order in a binary Ising lattice, Phys. Rev. 76, 1244 (1949).

[4] S. Sachdev, Quantum Phase Transitions (Cambridge University Press, Cambridge, 2007).

[5] R. Coldea, D. A. Tennant, E. M. Wheeler, E. Wawrzynska, D. Prabhakaran, M. Telling, K. Habicht, P. Smeibidl, and K. Kiefer, Quantum criticality in an Ising chain: Experimental evidence for emergent $E_{8}$ symmetry, Science 327, 177 (2010).

[6] A. Okutani, T. Kida, T. Usui, T. Kimura, K. Okunishi, and M. Hagiwara, High field magnetization of single crystals of the $S=1 / 2$ quasi-1D Ising-like antiferromagnet $\mathrm{SrCo}_{2} \mathrm{~V}_{2} \mathrm{O}_{8}$, Phys. Proc. 75, 779 (2015).

[7] Q. Faure, S. Takayoshi, S. Petit, V. Simonet, S. Raymond, L.-P. Regnault, M. Boehm, J. S. White, M. Mânsson, C. Rüegg, P. Lejay, B. Canals, T. Lorenz, S. C. Furuya, T. Giamarchi, and B. Grenier, Topological quantum phase transition in the Ising-like antiferromagnetic spin chain $\mathrm{BaCo}_{2} \mathrm{~V}_{2} \mathrm{O}_{8}$, Nat. Phys. 14, 716 (2018).

[8] M. Matsuda, H. Onishi, A. Okutani, J. Ma, H. Agrawal, T. Hong, D. M. Pajerowski, J. R. D. Copley, K. Okunishi, M. Mori, S. Kimura, and M. Hagiwara, Magnetic structure and dispersion relation of the $S=\frac{1}{2}$ quasi-one-dimensional Isinglike antiferromagnet $\mathrm{BaCo}_{2} \mathrm{~V}_{2} \mathrm{O}_{8}$ in a transverse magnetic field, Phys. Rev. B 96, 024439 (2017).

[9] Z. Wang, T. Lorenz, D. I. Gorbunov, P. T. Cong, Y. Kohama, S. Niesen, O. Breunig, J. Engelmayer, A. Herman, J. Wu, K. Kindo, J. Wosnitza, S. Zherlitsyn, and A. Loidl, Quantum Criticality of an Ising-Like Spin-1/2 Antiferromagnetic Chain in a Transverse Magnetic Field, Phys. Rev. Lett. 120, 207205 (2018).

[10] Z. Wang, J. Wu, W. Yang, A. K. Bera, D. Kamenskyi, A. T. M. Nazmul Islam, S. Xu, J. M. Law, B. Lake, C. $\mathrm{Wu}$, and A. Loidl, Experimental observation of Bethe strings, Nature (London) 554, 219 (2018).

[11] Z. Wang, M. Schmidt, A. Loidl, J. Wu, H. Zou, W. Yang, C. Dong, Y. Kohama, K. Kindo, D. I. Gorbunov, S. Niesen, O. Breunig, J. Engelmayer, and T. Lorenz, Quantum Critical Dynamics of a Heisenberg-Ising Chain in a Longitudinal Field: Many-Body Strings versus Fractional Excitations, Phys. Rev. Lett. 123, 067202 (2019).

[12] Y. Cui, H. Zou, N. Xi, Z. He, Y. X. Yang, L. Shu, G. H. Zhang, Z. Hu, T. Chen, R. Yu, J. Wu, and W. Yu, Quantum Criticality of the Ising-Like Screw Chain Antiferromagnet $\mathrm{SrCo}_{2} \mathrm{~V}_{2} \mathrm{O}_{8}$ in a Transverse Magnetic Field, Phys. Rev. Lett. 123, 067203 (2019).

[13] H. Liu and G. Khaliullin, Pseudospin exchange interactions in $d^{7}$ cobalt compounds: Possible realization of the Kitaev model, Phys. Rev. B 97, 014407 (2018).

[14] R. Sano, Y. Kato, and Y. Motome, Kitaev-Heisenberg Hamiltonian for high-spin $d^{7}$ Mott insulators, Phys. Rev. B 97, 014408 (2018).

[15] J. W. Krizan and R. J. Cava, $\mathrm{NaSrCo}_{2} \mathrm{~F}_{7}$, a $\mathrm{Co}^{2+}$ pyrochlore antiferromagnet, J. Phys.: Condens. Matter 27, 296002 (2015).

[16] B. A. Frandsen, K. A. Ross, J. W. Krizan, G. J. Nilsen, A. R. Wildes, R. J. Cava, R. J. Birgeneau, and S. J. L. Billinge,
Real-space investigation of short-range magnetic correlations in fluoride pyrochlores $\mathrm{NaCaCo}_{2} \quad \mathrm{~F}_{7}$ and $\mathrm{NaSrCo}_{2} \quad \mathrm{~F}_{7}$ with magnetic pair distribution function analysis, Phys. Rev. Mater. 1, 074412 (2017).

[17] K. A. Ross, J. M. Brown, R. J. Cava, J. W. Krizan, S. E. Nagler, J. A. Rodriguez-Rivera, and M. B. Stone, Single-ion properties of the $S_{\text {eff }}=\frac{1}{2} \mathrm{XY}$ antiferromagnetic pyrochlores $\mathrm{NaA}^{\prime} \mathrm{Co}_{2} \mathrm{~F}_{7}$ $\left(\mathrm{A}^{\prime}=\mathrm{Ca}^{2+}, \mathrm{Sr}^{2+}\right)$, Phys. Rev. B 95, 144414 (2017).

[18] F. A. Cevallos, K. Stolze, T. Kong, and R. J. Cava, Anisotropic magnetic properties of the triangular plane lattice material $\mathrm{TmMgGaO}_{4}$, Mater. Res. Bull. 105, 154 (2018).

[19] Y. Li, S. Bachus, Y. Tokiwa, A. A. Tsirlin, and P. Gegenwart, Absence of zero-point entropy in a triangular Ising antiferromagnet, arXiv:1804.00696.

[20] Y. Shen, C. Liu, Y. Qin, S. Shen, Y.-D. Li, R. Bewley, A. Schneidewind, G. Chen, and J. Zhao, Intertwined dipolar and multipolar order in the triangular-lattice magnet $\mathrm{TmMgGaO}_{4}$, Nat. Commun. 10, 4530 (2019).

[21] Z. L. Dun, J. Trinh, K. Li, M. Lee, K. W. Chen, R. Baumbach, Y. F. Hu, Y. X. Wang, E. S. Choi, B. S. Shastry, A. P. Ramirez, and H. D. Zhou, Magnetic Ground States of the Rare-Earth Tripod Kagome Lattice $\mathrm{Mg}_{2} \mathrm{RE}_{3} \mathrm{Sb}_{3} \mathrm{O}_{14}(\mathrm{RE}=\mathrm{Gd}$, Dy, Er), Phys. Rev. Lett. 116, 157201 (2016).

[22] Z. L. Dun, J. Trinh, M. Lee, E. S. Choi, K. Li, Y. F. Hu, Y. X. Wang, N. Blanc, A. P. Ramirez, and H. D. Zhou, Structural and magnetic properties of two branches of the tripod-kagomelattice family $\mathrm{A}_{2} \mathrm{R}_{3} \mathrm{Sb}_{3} \mathrm{O}_{14}(\mathrm{~A}=\mathrm{Mg}, \mathrm{Zn} ; \mathrm{R}=\mathrm{Pr}, \mathrm{Nd}, \mathrm{Gd}, \mathrm{Tb}$, Dy, Ho, Er, Yb), Phys. Rev. B 95, 104439 (2017).

[23] J. A. M. Paddison, H. S. Ong, J. O. Hamp, P. Mukherjee, X. Bai, M. G. Tucker, N. P. Butch, C. Castelnovo, M. Mourigal, and S. E. Dutton, Emergent order in the kagome Ising magnet $\mathrm{Dy}_{3} \mathrm{Mg}_{2} \mathrm{Sb}_{3} \mathrm{O}_{14}$, Nat. Commun. 7, 13842 (2016).

[24] Z.-F. Ding, Y.-X. Yang, J. Zhang, C. Tan, Z.-H. Zhu, G. Chen, and L. Shu, Possible gapless spin liquid in the rareearth kagome lattice magnet $\mathrm{Tm}_{3} \mathrm{Sb}_{3} \mathrm{Zn}_{2} \mathrm{O}_{14}$, Phys. Rev. B 98, 174404 (2018).

[25] E. Lhotel, S. Petit, M. Ciomaga Hatnean, J. Ollivier, H. Mutka, E. Ressouche, M. R. Lees, and G. Balakrishnan, Evidence for dynamic kagome ice, Nat. Commun. 9, 3786 (2018).

[26] T. Fennell, S. T. Bramwell, D. F. McMorrow, P. Manuel, and A. R. Wildes, Pinch points and Kasteleyn transitions in kagome ice, Nat. Phys. 3, 566 (2007).

[27] K. Matsuhira, Z. Hiroi, T. Tayama, S. Takagi, and T. Sakakibara, A new macroscopically degenerate ground state in the spin ice compound $\mathrm{Dy}_{2} \mathrm{Ti}_{2} \mathrm{O}_{7}$ under a magnetic field, J. Phys.: Condens. Matter 14, L559 (2002).

[28] P. Nikolić and T. Senthil, Theory of the kagome lattice Ising antiferromagnet in weak transverse fields, Phys. Rev. B 71, 024401 (2005).

[29] T. Fennell, P. P. Deen, A. R. Wildes, A. R. Schmalzl, D. Prabhakaran, A. T. Boothroyd, R. J. Aldus, D. F. McMorrow, and S. T. Bramwell, Magnetic coulomb phase in the spin ice $\mathrm{Ho}_{2} \mathrm{Ti}_{2} \mathrm{O}_{7}$, Science 326, 415 (2009).

[30] Z. Dun, X. Bai, J. A. M. Paddison, N. P. Butch, C. D. Cruz, M. B. Stone, T. Hong, M. Mourigal, and H. Zhou, Quantum spin fragmentation in kagome ice $\mathrm{Ho}_{3} \mathrm{Mg}_{2} \mathrm{Sb}_{3} \mathrm{O}_{14}$, arXiv:1806.04081.

[31] R. Moessner, S. L. Sondhi, and P. Chandra, Two-Dimensional Periodic Frustrated Ising Models in a Transverse Field, Phys. Rev. Lett. 84, 4457 (2000). 
[32] R. Moessner and S. L. Sondhi, Ising models of quantum frustration, Phys. Rev. B 63, 224401 (2001).

[33] S. Biswas, G. Rakala, and K. Damle, Quantum cluster algorithm for frustrated Ising models in a transverse field, Phys. Rev. B 93, 235103 (2016).

[34] S. Biswas and K. Damle, Singular ferromagnetic susceptibility of the transverse-field Ising antiferromagnet on the triangular lattice, Phys. Rev. B 97, 085114 (2018).

[35] R. Moessner and S. L. Sondhi, Theory of the [111] magnetization plateau in spin ice, Phys. Rev. B 68, 064411 (2003).

[36] R. Moessner, S. L. Sondhi, and P. Chandra, Phase diagram of the hexagonal lattice quantum dimer model, Phys. Rev. B 64, 144416 (2001).

[37] SungBin Lee, S. Onoda, and L. Balents, Generic quantum spin ice, Phys. Rev. B 86, 104412 (2012).

[38] B. I. Halperin, T. C. Lubensky, and S.-K. Ma, First-Order Phase Transitions in Superconductors and Smectic- $A$ Liquid Crystals, Phys. Rev. Lett. 32, 292 (1974).

[39] H. Li, Y.-D. Liao, B.-B. Chen, X.-T. Zeng, X.-L. Sheng, Y. Qi, Z. Y. Meng, and W. Li, The ghost of a vanishing stripe order in the triangular quantum Ising magnet $\mathrm{TmMgGaO}_{4}$, arXiv:1907.08173.
[40] J. Koziol, S. Fey, S. C. Kapfer, and K. P. Schmidt, Quantum criticality of the transverse-field Ising model with long-range interactions on triangular-lattice cylinders, Phys. Rev. B 100, 144411 (2019).

[41] R. K. Kaul and A. W. Sandvik, Lattice Model for the SU(N) Néel to Valence-Bond Solid Quantum Phase Transition at Large N, Phys. Rev. Lett. 108, 137201 (2012).

[42] M. S. Block and R. K. Kaul, Néel-VBS transition in threedimensional SU $(N)$ antiferromagnets, Phys. Rev. B 86, 134408 (2012).

[43] R. K. Kaul, R. G. Melko, and A. W. Sandvik, Bridging latticescale physics and continuum field theory with quantum Monte Carlo simulations, Annu. Rev. Condens. Matter Phys. 4, 179 (2013).

[44] M. R. Norman, Colloquium: Herbertsmithite and the search for the quantum spin liquid, Rev. Mod. Phys. 88, 041002 (2016).

[45] D. I. Khomskii, Electric dipoles on magnetic monopoles in spin ice, Nat. Commun. 3, 904 (2012).

[46] F.-Y. Li, Y.-D. Li, Y. Yu, A. Paramekanti, and G. Chen, Kitaev materials beyond iridates: Order by quantum disorder and Weyl magnons in rare-earth double perovskites, Phys. Rev. B 95, 085132 (2017). 